\title{
Undiagnosed and Misdiagnosed Chronic Obstructive Pulmonary Disease: Data from the BOLD Australia Study
}

This article was published in the following Dove Press journal: International Journal of Chronic Obstructive Pulmonary Disease

\author{
Kate Petrie' \\ Brett G Toelle (iD ${ }^{2,3}$ \\ Richard Wood-Baker ${ }^{4}$ \\ Graeme P Maguire (D ${ }^{5}$ \\ Alan L James ${ }^{6,7}$ \\ Michael Hunter iD $^{8,9}$ \\ David P Johns (iD) \\ Guy B Marks 2,10 \\ Johnson George ${ }^{1,11}$ \\ Michael J Abramson (iD ${ }^{\prime \prime}$ \\ 'Centre for Medicine Use and Safety, Faculty \\ of Pharmacy and Pharmaceutical Sciences, \\ Monash University, Melbourne, VIC, \\ Australia; ${ }^{2}$ Woolcock Institute of Medical \\ Research, The University of Sydney, Sydney, \\ NSW, Australia; ${ }^{3}$ Sydney Local Health \\ District, Sydney, NSW, Australia; ${ }^{4}$ College of \\ Health and Medicine, University of Tasmania, \\ Hobart, TAS, Australia; ${ }^{5}$ Western Clinical \\ School, University of Melbourne, \\ Melbourne, Australia and General Internal \\ Medicine, Western Health, Melbourne, VIC, \\ Australia; ${ }^{6}$ Sir Charles Gairdner Hospital, \\ West Australian Sleep Disorders Research \\ Institute, Department of Pulmonary \\ Physiology and Sleep Medicine, Perth, WA, \\ Australia; ${ }^{7}$ University of Western Australia, \\ Medical School, Perth, WA, Australia; \\ ${ }^{8}$ School of Population and Global Health, \\ University of Western Australia, Perth, WA, \\ Australia; ${ }^{\circ}$ Busselton Population Medical \\ Research Institute, Busselton, WA, Australia; \\ ${ }^{10}$ South Western Sydney Clinical School, \\ University of New South Wales, Sydney, \\ NSW, Australia; " School of Public Health \\ and Preventive Medicine, Monash University, \\ Melbourne, VIC, Australia
}

Correspondence: Michael J Abramson School of Public Health and Preventive Medicine, Monash University, Level 2, 553

St Kilda Road, Melbourne, VIC, 3004, Australia

Tel +6I 399030573

Fax +6I 399030556

Email michael.abramson@monash.edu
Purpose: Spirometry is necessary to confirm COPD, but many patients are diagnosed based on clinical presentation and/or chest x-ray. There are also those who do not present to primary care for case finding and remain undiagnosed. We aimed to identify: (a) factors that are associated with undiagnosed COPD; and (b) factors that are associated with a potential misdiagnosis of COPD.

Patients and Methods: This analysis used data from the Burden of Obstructive Lung Disease (BOLD), a cross-sectional study of community dwelling adults randomly selected from six study sites, chosen to provide a representative sample of the Australian population $(n=3357)$. Participants were grouped by COPD diagnostic criteria based on spirometry and self-reported diagnosis. Odds ratios for predictors of undiagnosed and misdiagnosed were estimated using logistic regression.

Results: Of the BOLD Australia sample, 1.8\% had confirmed COPD, of whom only half self-reported a diagnosis of COPD. A further $6.9 \%$ probably had COPD, but were undiagnosed. The priority target population for case finding of undiagnosed COPD was aged $\geq 60$ years (particularly those $\geq 75$ years), with wheezing, shortness of breath and a body mass index (BMI) $<25 \mathrm{~kg} / \mathrm{m}^{2}$. The priority target population for identifying and reviewing misdiagnosed COPD was aged $<60$ years, female, with no wheezing and a BMI $\geq 25 \mathrm{~kg} / \mathrm{m}^{2}$.

Conclusion: Challenges continue in accurately diagnosing COPD and greater efforts are needed to identify undiagnosed and misdiagnosed individuals to ensure an accurate diagnosis and the initiation of appropriate management in order to reduce the burden of COPD.

Keywords: case finding, diagnosis, spirometry, epidemiology

\section{Introduction}

Chronic obstructive pulmonary disease (COPD) was the third leading cause of death worldwide in 2017. ${ }^{1}$ Therefore, preventive and management strategies need to be implemented to reduce this burden of disease. In Australia, the COPD-X Plan (Australia and New Zealand guidelines for the management of COPD), recommend a case finding approach to enable an earlier diagnosis of COPD. ${ }^{2}$ Case finding, in the Australian context, is defined as "the examination of an individual or group suspected as having, or at risk of, the condition". 3

There will be patients who do not present to primary care with respiratory symptoms, so they will not participate in a case finding review and will continue to remain undiagnosed. ${ }^{4}$ Remaining undiagnosed and untreated for COPD, potentially impacts on the patient's quality of life and disease progression in those who 
experience preventable exacerbations. Remaining undiagnosed may also impact on the opportunities for support and interventions for smoking cessation for those who continue to smoke.

Along with case finding those previously undiagnosed with COPD, there are also many patients diagnosed based on clinical presentation and/or chest x-ray, without spirometry, potentially leading to misdiagnosis. ${ }^{5,6}$ In order to confirm COPD, spirometry is necessary. A misdiagnosis of COPD may result in patients being over-treated with costly and potentially inappropriate medications and may divert attention from the underlying cause(s) of their symptoms. Misdiagnosis may, in addition, impact patient quality of life as they have been (incorrectly) diagnosed with a progressive chronic illness that has no cure. It is, therefore, imperative to understand and address the factors that contribute to being either mis- or undiagnosed, so more accurate diagnoses can be made to improve quality of life, reduce the frequency of exacerbations, support smoking cessation, improve quality of life and ultimately aim to reduce the burden of COPD.

The aims of this analysis were to identify: (a) factors that are associated with undiagnosed COPD; and (b) factors that are associated with a potential misdiagnosis of COPD.

\section{Methods}

\section{Sample}

This analysis used data from BOLD Australia, which recruited non-institutionalised adults aged $\geq 40$ years who were randomly selected from the electoral rolls in New South Wales, Victoria and Tasmania. ${ }^{7}$ Participants in Western Australia (WA) were recruited from census data in Broome or randomly from within the Busselton Health Study. ${ }^{7}$ The 12,526 individuals in the initial study sample were contacted by mail to participate and invited to telephone for an appointment. ${ }^{7}$ After two weeks, potential participants were contacted by telephone as a follow-up and invited to participate. ${ }^{7}$ Further attempts to contact nonresponders were undertaken via telephone and mail. ${ }^{7}$ Data collection was undertaken between 2006 and $2010{ }^{7}$ Data collection at each site was completed within 12 months, except for The Kimberley (WA) where data collection continued for four years due to the remoteness of the sites. Following previously published exclusion criteria (such as participants who were not contactable, $<40$ years old, institutionalised, or unable to perform spirometry, refused, moved out of the area), the sample for this analysis comprised 3357 individuals (Supplementary Figure S1). ${ }^{7}$ More detailed information of sample selection has been published elsewhere. ${ }^{7}$

\section{Description of Variables}

All participants completed the BOLD core questionnaire that included details of smoking status, occupational exposures, respiratory symptoms, inhaler use and demographics (Supplementary information S1). ${ }^{7}$ Specific comorbidities reported were asthma, hypertension, heart disease, diabetes, lung cancer and stroke. Moderate or severe exacerbation risk was assessed, as either high or low, using the GOLD 2019 definition. "High" was at least two presentations (to primary care) with worsening symptoms or at least one episode leading to hospital admission and "low" a maximum of one presentation (to primary care) with worsening symptoms. ${ }^{8}$ Socioeconomic status was reported as quintiles of Socio-Economic Indexes for Areas (SEIFA) from the Australian Bureau of Statistics (ABS) Census 2011, with one being the "most disadvantaged" and five being the "least disadvantaged". Rurality was categorised as residing in major cities, inner regional, outer regional, remote or very remote areas of Australia.

Quality of life was reported as domain scores of the Short-Form (SF)-12 questionnaire. ${ }^{9}$ Computed physical and mental component summary scores (PCS, MCS) of SF-12 used US norms as no Australian norms were available. ${ }^{9}$ All study participants performed postbronchodilator spirometry, according to the American Thoracic Society/European Respiratory Society standards, using the EasyOne spirometer (ndd Medizintechnik, Zürich, Switzerland) and all spirograms were reviewed for quality. ${ }^{7,10}$ The criteria for defining the presence of airflow obstruction, based on the value of the forced expiratory volume in 1 second $\left(\mathrm{FEV}_{1}\right)$ and forced vital capacity (FVC) ratio $\left(\mathrm{FEV}_{1} / \mathrm{FVC}\right)$, were: (1) a fixed cutoff value (less than 0.70 ) or (2) the lower limit of normal (LLN), defined as the 5th percentile of the distribution. The LLN was calculated using the Global Lung Initiative (GLI-2012) tool, available from the European Respiratory Society. ${ }^{11}$

\section{Spirometrically Defined Diagnostic Groups}

Post-bronchodilator spirometry and self-reported diagnoses were used to group participants. Due to the lack of 
a "gold standard" criteria for defining COPD, we have defined COPD as those that met both the fixed cut-off $\left(\mathrm{FEV}_{1} / \mathrm{FVC}\right.$ ratio $\left.<0.70\right)$ and LLN criteria. The definitions of each group were as follows: ${ }^{12}$

- Confirmed diagnosis: Self-reported COPD diagnosis, post-bronchodilator $\mathrm{FEV}_{1} / \mathrm{FVC}$ below fixed cut-off and below LLN.

- Probably undiagnosed: Post-bronchodilator FEV 1 /FVC below fixed cut-off and below LLN. No selfreported COPD diagnosis.

- Probably misdiagnosed: Self-reported COPD diagnosis. Neither fixed cut-off nor LLN criteria met.

\section{Data Analysis}

Data analyses were conducted using Stata version $15.1^{13}$ with $\mathrm{p}$-values less than 0.05 being considered statistically significant. The degree of overlap among all diagnostic groups was displayed graphically using an Euler diagram designed using eulerAPE. ${ }^{14}$ The distribution of demographic characteristics was examined overall and compared across the normal lung function, un-, mis- and confirmed diagnostic groups using Kruskal-Wallis tests.

In order to identify factors that might assist health professionals in identifying those with un- and misdiagnosed COPD, the undiagnosed group was compared to those with normal lung function and the misdiagnosed group was compared to those with a confirmed diagnosis of COPD. Multivariate logistic regression models were used to calculate adjusted odds ratios (OR) with 95\% confidence intervals (CI). The inclusion of variables was guided by published literature, consideration of practicalities (that is, relative ease in identifying the patient factor(s) by health professionals) and included age, gender, smoking status, respiratory symptoms (cough, phlegm, wheeze and shortness of breath [SOB]) and BMI., Potential differences between recruitment sites (Supplementary Table S1) were not considered in the model, as we aimed to produce single target populations (for un- and misdiagnosed COPD) for use Australia wide. Sensitivity analyses were also performed if diagnosis by fixed cut-off only and also excluding participants with self-reported asthma (Supplementary Figure S2, Tables $\underline{\mathrm{S} 2}-\underline{5}$ ). Receiver operating characteristic (ROC) curves were produced to assess the utility of the regression models ( $\underline{\text { Supplementary Figures S3 }}$ and S4).

\section{Ethical Review}

This study was approved by the respective ethics committees of the participating universities and conformed to the Declaration of Helsinki. Each study site obtained informed participant consent as per site specific ethics approvals. Ethics approval for this analysis was received from Monash University Human Ethics Committee (application number 12000).

\section{Results}

\section{Participant Characteristics}

Of the 3357 participants included in the BOLD Australia sample, two were excluded from receiving a diagnostic group, as one had missing spirometry and one had missing data on ethnicity. One participant was aged 97 years and as the GLI-2012 reference equations have a maximum of 95 years of age, we set the age to 95 years for this case in the GLI-2012 tool. $^{11}$

The majority of participants had normal lung function $(n=2736,81.5 \%)$. Only 60 participants $(1.8 \%)$ had a selfreported diagnosis of COPD and met both the fixed cut-off and LLN definition of airflow obstruction. The degree of overlap between all diagnostic groups is presented in Figure 1.

Of the 3355 participants, 233 (6.9\%) had probably undiagnosed COPD, meeting both the fixed cut-off and LLN, but did not self-report a diagnosis of COPD (Table 1) and might therefore benefit from case finding review. When compared to those with normal lung function, the undiagnosed were older, more likely to be ever smokers, male, had lower mean percent predicted (GLI) $\mathrm{FEV}_{1}$, a higher prevalence of all respiratory symptoms, more inhaler use, a lower BMI and also had worse quality of life (lower SF-12 PCS and MCS) (Table 1).

There were $74(2.2 \%)$ participants who had a selfreported COPD diagnosis, but did not meet either the fixed cut-off or LLN definition of COPD. These probably misdiagnosed, when compared to those with a confirmed diagnosis, were younger, more likely to be never smokers, female, had higher mean percent predicted (GLI) $\mathrm{FEV}_{1}$, a lower prevalence of respiratory symptoms and a higher BMI (Table 1). Those with BMI $\geq 25 \mathrm{~kg} / \mathrm{m}^{2}$ and BMI $\geq 30 \mathrm{~kg} / \mathrm{m}^{2}$ corresponded to approximately $50 \%$ and $18 \%$ of the confirmed diagnosis group compared to $67 \%$ and $36 \%$ of the misdiagnosed group.

Further characteristics across diagnostic groups and comparison between recruitment sites can be seen in

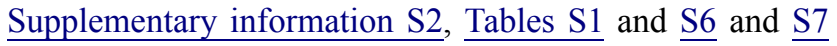




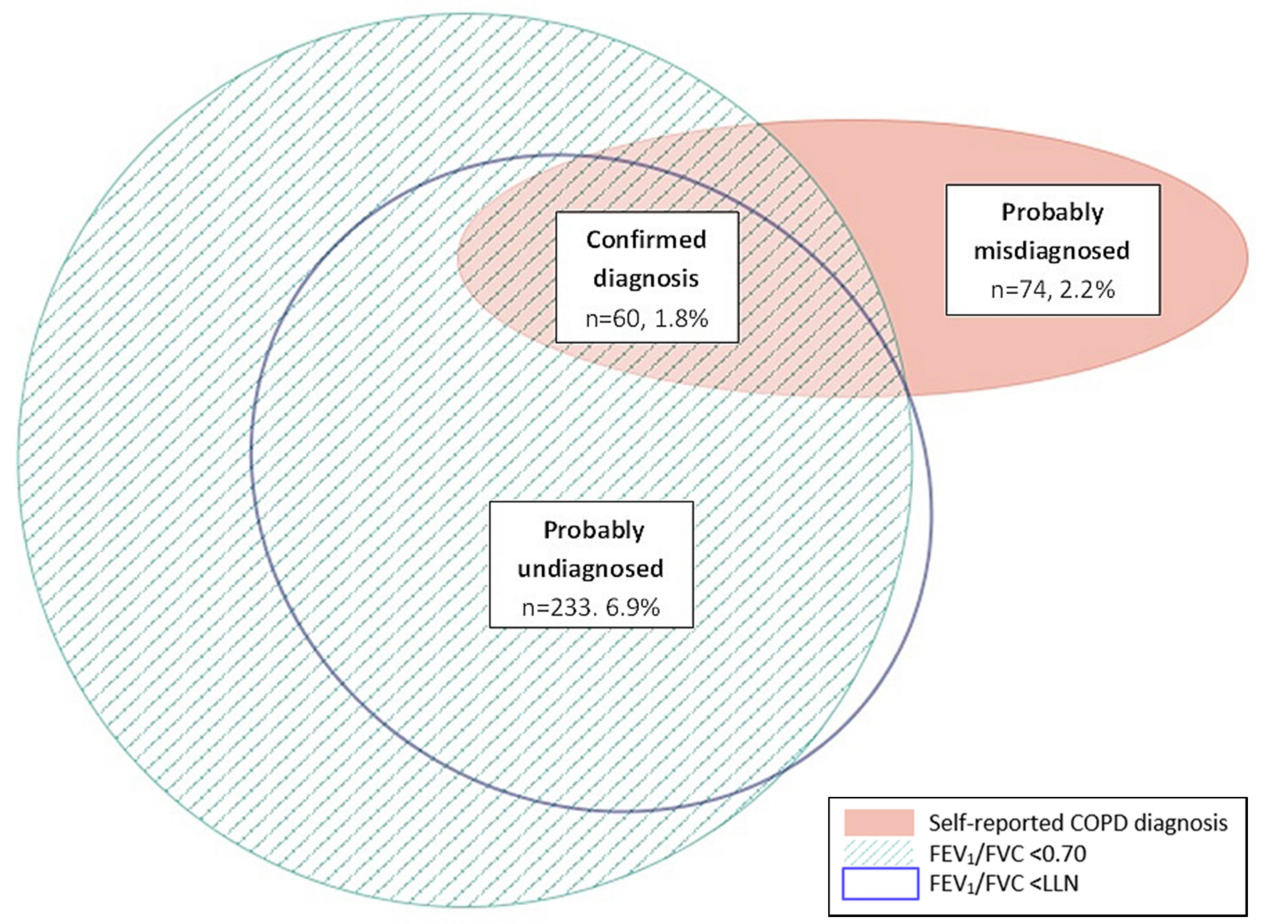

Figure I Euler diagram showing proportion of undiagnosed, misdiagnosed and confirmed COPD in the sample.

and Figure S3. And sensitivity analyses using fixed cut-off only and excluding those with known asthma can be seen in Supplementary Tables S2 $-\underline{5}$ and Figure S2.

\section{Target Population for Case Finding and Diagnostic Review}

The predictors of probably undiagnosed COPD (versus normal lung function) and probably misdiagnosed COPD (versus a confirmed diagnosis) are shown in Tables 2 and 3. Compared to those aged less than 60 years, those older than 60 had higher odds of having undiagnosed COPD. Reporting wheeze was the strongest predictor of having undiagnosed COPD. Other predictors of undiagnosed COPD were being an ever smoker, SOB and BMI $<25 \mathrm{~kg} / \mathrm{m}^{2}$. Predictors of probably misdiagnosed COPD were absence of wheeze and BMI of $\geq 25 \mathrm{~kg} / \mathrm{m}^{2}$. Females also had higher odds of being misdiagnosed. Those aged 60 years or over had lower odds of being misdiagnosed compared to those under 60 years.

When using the fixed cut-off and self-reported diagnosis of COPD only, age is a much stronger predictor of undiagnosed COPD as, unlike the use of the LLN definition, the fixed cut-off does not take age into account (Supplementary Tables S2 and S3).
When participants with asthma were removed from the logistic regression models, in general, the coefficients were robust (Tables S4 and $\underline{\mathrm{S} 5}$ ). In addition, in most cases, statistical significance of the covariates remained similar although in some cases, likely due to a reduction in statistical power, the p-values increased in the undiagnosed model (Table S4). After removing participants with asthma, for those probably misdiagnosed, females, and those without SOB, had statistically significant greater odds of being misdiagnosed with COPD (Table S5).

\section{Discussion}

Of the BOLD Australia sample who self-reported a diagnosis of COPD, one half had probably been misdiagnosed and less than one half had a spirometrically confirmed diagnosis. Approximately one in 15 participants were probably undiagnosed and met both the fixed cut-off and LLN criteria. Given the burden of COPD, there need to be efforts to increase case finding approaches in undiagnosed cases, and a diagnostic review of those who selfreport COPD to identify those who have probably been misdiagnosed.

To ensure that health professionals can target appropriate patients, the undiagnosed were compared to those with normal lung function and the misdiagnosed were 


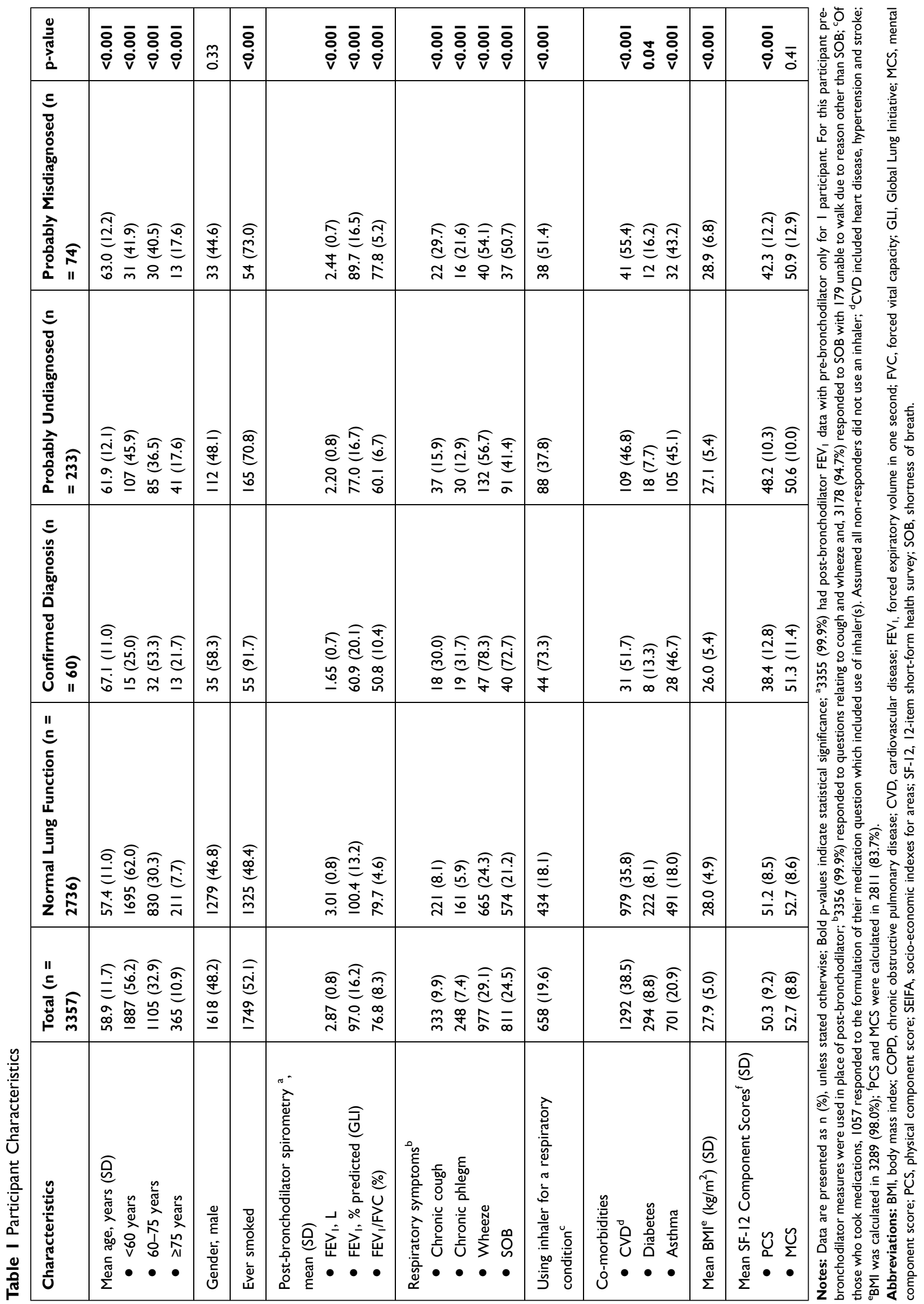


Table 2 Predictors of Probably Undiagnosed COPD (versus Normal Lung Function)

\begin{tabular}{|c|c|c|c|}
\hline & OR & 95\% Cl & p-value \\
\hline Age & & & \\
\hline - <60 years (reference) & 1.00 & & \\
\hline - 60-75 years & 2.07 & I.49-2.87 & $<0.001$ \\
\hline$\bullet \geq 75$ years & 3.51 & $2.20-5.59$ & $<0.001$ \\
\hline Gender, male & 1.27 & $0.93-1.72$ & 0.13 \\
\hline Ever smoker & 2.39 & $1.73-3.30$ & $<0.001$ \\
\hline Chronic cough & 1.31 & $0.82-2.10$ & 0.11 \\
\hline Chronic phlegm & 1.24 & $0.73-2.08$ & 0.42 \\
\hline Wheeze & 4.01 & $2.94-5.47$ & $<0.001$ \\
\hline SOB & 2.27 & $1.64-3.67$ & $<0.001$ \\
\hline $\mathrm{BMI}<25 \mathrm{~kg} / \mathrm{m}^{2}$ & 2.66 & $1.92-3.67$ & $<0.001$ \\
\hline
\end{tabular}

Note: Bold p-values indicate statistical significance.

Abbreviations: $\mathrm{BMI}$, body mass index; $\mathrm{Cl}$, confidence intervals; OR, odds ratio; SOB, shortness of breath.

Table 3 Predictors of Probably Misdiagnosed COPD (versus Confirmed Diagnosis of COPD)

\begin{tabular}{|c|c|c|c|}
\hline & OR & $95 \% \mathrm{Cl}$ & p-value \\
\hline \multirow{4}{*}{$\begin{array}{l}\text { Age } \\
\text { - }<60 \text { years (reference) } \\
\text { - } 60-75 \text { years } \\
\text { - } \geq 75 \text { years }\end{array}$} & & & \\
\hline & 1.00 & & \\
\hline & 0.42 & $0.13-1.32$ & 0.14 \\
\hline & 0.42 & $0.10-1.68$ & 0.22 \\
\hline Gender, male & 0.33 & $0.12-0.90$ & 0.03 \\
\hline Ever smoker & 0.28 & $0.07-1.13$ & 0.07 \\
\hline Chronic cough & 1.69 & $0.55-5.18$ & 0.36 \\
\hline Chronic phlegm & 0.65 & $0.22-1.94$ & 0.44 \\
\hline Wheeze & 0.19 & $0.07-0.52$ & 0.001 \\
\hline SOB & 0.34 & $0.11-1.01$ & 0.05 \\
\hline $\mathrm{BMI}<25 \mathrm{~kg} / \mathrm{m}^{2}$ & 0.31 & $0.11-0.85$ & 0.02 \\
\hline
\end{tabular}

Note: Bold $\mathrm{p}$-values indicate statistical significance.

Abbreviations: $\mathrm{BMI}$, body mass index; $\mathrm{Cl}$, confidence intervals; OR, odds ratio; SOB, shortness of breath.

compared to those with a confirmed diagnosis. Those with undiagnosed COPD may be most easily identified as older (especially over 75 years), with symptoms and normal BMI. These characteristics are comparable to those found in a recent pooled cohort study $(\mathrm{n}=6710$; mean age 58 years) that aimed to determine the prevalence of COPD in an analysis of former and current smokers in
Denmark. $^{15}$ People with these specific characteristics should be targeted in primary care and encouraged to participate in case finding review. Those with undiagnosed COPD also had lower levels of self-reported inhaler use, indicating that patients were not receiving treatment even in the presence of respiratory symptoms.

Those probably misdiagnosed with COPD may be most easily identified as females under 60 years of age, without wheeze but overweight/obese. Health professionals should ensure that patients with a documented history of COPD and these specific characteristics have the diagnosis objectively confirmed by post-bronchodilator spirometry to minimise misdiagnosis. A misdiagnosis of COPD may divert focus from the underlying cause(s) of the patient's symptoms, such as cardiovascular disease or obesity, and expose patients inappropriately to costly and ineffective treatments. Previous studies have highlighted the need for an increase in the utilisation of spirometry in primary care, and our study further supports this need. ${ }^{5,6,16}$ From our analyses and other literature, some symptoms may be due to obesity, increasing misdiagnosis amongst middle aged people who are overweight or obese. ${ }^{17,18}$ Given that approximately two-thirds of the Australian adult population were overweight or obese in 2017-18, it may be particularly relevant to review the COPD diagnosis in this group. ${ }^{19}$

The prevalence of misdiagnosed COPD found in our study was comparable to other studies. A recent randomised controlled trial that recruited participants with a smoking history, found that of patients managed in primary care with COPD, about one half had airflow obstruction (using fixed cut-off) and/or clinical findings consistent with COPD. ${ }^{20}$ Case finding and spirometry were able to identify a substantial number of participants with undiagnosed COPD. ${ }^{20}$ Our study confirmed these findings, but also included participants without a history of smoking, and used both the fixed cut-off or LLN method for confirming COPD, overcoming limitations of either method.

Wheeze was one symptom that predicted both undiagnosed COPD and misdiagnosed COPD, despite there being no statistically significant difference in the proportions of participants with asthma in each of these groups (Table 1, $\underline{\text { Supplementary Tables S4 }}$ and S5). A recent study that used respiratory symptoms to predict mortality in a cohort of older people, found that current smokers with wheeze had the shortest life expectancy compared to those with differing symptoms and smoking status. ${ }^{21}$ An 
increased focus on patients who present to health professionals with wheezing may aid in identifying those with undiagnosed COPD, and those with other respiratory conditions. ${ }^{22}$

One of the main strengths of our study was that comparisons were made between different diagnostic groups, including those with normal lung function, enabling a case finding target population to be defined. Other studies have only considered the patient characteristics associated with different COPD diagnostic groups. A large proportion of these were cohort studies that included only patients with COPD (defined by various methods), preventing comparison with patients with normal lung function. ${ }^{6,15,23-25}$ Other strengths included that data were collected following a standardised international protocol, the nationwide sample, large sample size and the use of both the fixed cutoff and LLN methods for determining airflow obstruction.

The criteria for diagnosing COPD are still debated, both in Australia and internationally, but there is agreement that investigation should include post-bronchodilator spirometry with estimation of the $\mathrm{FEV}_{1} / \mathrm{FVC}$ ratio as an index of airflow obstruction. ${ }^{12,26}$ The choice of criteria, between fixed cut-off and LLN, has an impact on the diagnosis of COPD. These analyses include both criteria to overcome the known limitations of each - that using the fixed cut-off results in over-diagnoses of COPD in older people and that using the LLN results in over-diagnoses in younger people. ${ }^{27,28}$ Regardless of whether the fixed cutoff or LLN is used, the undiagnosed and the misdiagnosed groups could be targeted by health professionals to reduce the burden of COPD.

However, our study also had some limitations. The diagnostic groupings used were based on spirometry and self-reported COPD diagnosis. The groupings did not include an assessment of respiratory symptoms and/or other risk factors, as it could not be determined if the symptom(s) would have been reported to primary care health professionals. ${ }^{8}$ Despite the large sample size, some diagnostic groups had relatively few participants and therefore only a modest number of co-variates could be included in the analysis to determine predictors of unand misdiagnosed COPD without significant increases to the uncertainty around the estimates obtained. Participants were also not a truly random sample of the Australian population, as study sites were not selected completely at random, although previous work used posthoc weights to estimate prevalence rates for the Australian population. ${ }^{7}$ The overall response rate was low, potentially introducing a degree of selection bias, with those included in the analysis being younger and more likely to self-report a diagnosis of COPD than those who only reported minimal data. ${ }^{7}$ The selfreported diagnosis of COPD in the BOLD Australia sample was however comparable to the prevalence of COPD in the 2017-18 ABS National Health Survey (4.4\% and $4.8 \%$ respectively). ${ }^{29}$

More recent data with longitudinal analysis, linking lung function, symptoms, rurality, socioeconomic and other determinants of health, to outcomes such as exacerbations, quality of life and mortality will be valuable to identify targets and design interventions to reduce the burden of COPD. ${ }^{30,31}$ Further research into the outcomes of patients who have undiagnosed or misdiagnosed COPD (and compared with cases of confirmed COPD), would also be valuable.

\section{Conclusion}

One half of the BOLD Australia participants who reported a diagnosis of COPD had probably been misdiagnosed and significant numbers probably had undiagnosed COPD. Increased efforts by health professionals are needed to identify these un- and misdiagnosed individuals to ensure more accurate diagnoses are made and appropriate management and/or risk reduction strategies are offered. The need for increased and appropriate utilisation of spirometry in primary care is not new, but the ongoing need should be reinforced to all health professionals. This analysis provides potential criteria for identifying patients with undiagnosed and misdiagnosed COPD via targeted case finding and review in primary care, especially in patients with COPD-like symptoms that may be due to other conditions. Further longitudinal research linking lung function, symptoms and other determinants of health to outcomes such as exacerbations, quality of life and mortality would be valuable in reducing the global burden of COPD.

\section{Abbreviations}

ABS, Australian Bureau of Statistics; BMI, body mass index; BOLD, Burden of Obstructive Lung Disease; CI, confidence interval; COPD, chronic obstructive lung disease; CVD, cardiovascular disease; ERS, European Respiratory Society; $\mathrm{FEV}_{1}$, forced expiratory volume in one second; FVC, forced vital capacity; GLI, Global Lung Initiative; GOLD, Global Initiative for Chronic Obstructive Lung Disease; LLN, lower limit of normal; 
MCS, mental component score; NSW, New South Wales; OR, odds ratio; PCS, physical component score; ROC, receiver operating characteristic; $\mathrm{SD}$, standard deviation; SEIFA, Socio-Economic Indexes for Areas; SOB, shortness of breath; WA, Western Australia.

\section{Acknowledgments}

We wish to thank the participants in the BOLD Australia without whom the present study would not have been possible. The authors gratefully acknowledge the work of the research staff at each study site for recruiting and testing participants: Busselton: Elspeth Inglis, Peta Grayson; Broome: David Reeve, Matthew Yap, Mary Lane, Wendy Cavilla; Melbourne: Angela Lewis, Joan Raven, Joan Green; Rural New South Wales: Melanie Heine, Cassanne Eccleston, Julie Cooke, Brian Spurrell, Robyn Paton; Sydney: Kate Hardaker, Paola Espinel; Tasmania: Carol Phillips. KP acknowledges the support of the Australian Government Research Training Program Scholarship.

\section{Disclosure}

GPM is an advisor to Pfizer on smoking cessation strategies. GBM has provided independent medical service on an advisory board for Astra Zeneca and reports funds from GSK Australia to his institution. JG holds investigatorinitiated grants for unrelated research from Pfizer, GSK and Boehringer Ingelheim. He has received honoraria from a consultancy for GSK and for invited presentations at a continuing education event organised by Pfizer. MJA holds investigator-initiated grants for unrelated research from Pfizer and Boehringer Ingelheim. He has also undertaken an unrelated consultancy for and received assistance with conference attendance from Sanofi and received a speaker fee from GSK. GBM, MJA, RWB, GPM and ALJ report investigator initiated competitive research grant from NHMRC. The authors report no other conflicts of interest in this work.

\section{References}

1. Global Burden of Disease Collaborative Network. Global Burden of Disease Study 2017 (GBD 2017) Cause-Specific Mortality 1980-2017. Seattle: Institute for Health Metrics and Evaluation (IHME); 2018.

2. Yang I, Dabscheck E, George J, et al. The COPD-X plan: australian and New Zealand Guidelines for the management of chronic obstructive pulmonary disease. Thorac Soc Aust New Zeal Lung Found Aust. 2016.

3. Royal Australian College of General Practitioners (RACGP). Guidelines for Preventive Activities in General Practice. 9th ed. 2016.
4. Johnson KM, Bryan S, Ghanbarian S, Sin DD, Sadatsafavi M. Characterizing undiagnosed chronic obstructive pulmonary disease: a systematic review and meta-analysis. Respir Res. 2018;19(1):26. doi:10.1186/s12931-018-0731-1

5. Abramson MJ, Schattner RL, Sulaiman ND, Del Colle EA, Aroni R, Thien F. Accuracy of asthma and COPD diagnosis in Australian general practice: a mixed methods study. Prim Care Respir J. 2012;21:167-173. doi:10.4104/pcrj.2011.00103

6. Zwar NA, Marks GB, Hermiz O, et al. Predictors of accuracy of diagnosis of chronic obstructive pulmonary disease in general practice. Med J Aust. 2011;195(4):168-171. doi:10.5694/j.13265377.2011.tb03271.x

7. Toelle BG, Xuan W, Bird TE, et al. Respiratory symptoms and illness in older Australians: the burden of obstructive lung disease (BOLD) study. Med J Aust. 2013;198(3):144-148. doi:10.5694/mja11.11640

8. Global Initiative for Chronic Obstructive Lung Disease. Global strategy for the diagnosis, management, and prevention of chronic obstructive pulmonary disease. Published 2019. Available from: https://goldcopd.org/wp-content/uploads/2018/11/GOLD-2019-v1. 7-FINAL-14Nov2018-WMS.pdf. Accessed August 26, 2019.

9. Ware JE, Kosinski M, Turner-Bowker DM, Gandeck B. User's Manual for the SF-12v2tm Health Survey:(With a Supplement Documenting SF-12 Health Survey). RI: QualityMetric Incorporated Lincoln; 2007.

10. Buist AS, Vollmer WM, Sullivan SD, et al. The burden of obstructive lung disease initiative (BOLD): rationale and design. COPD J Chronic Obstr Pulm Dis. 2005;2(2):277-283. doi:10.1081/COPD57610

11. Quanjer PH, Stanojevic S, Cole TJ, et al. Multi-ethnic reference values for spirometry for the 3-95-yr age range: the global lung function 2012 equations. Eur Respir J. 2012;40(6):1324-1343. doi:10.1183/09031936.00080312

12. Diab N, Gershon AS, Sin DD, et al. Underdiagnosis and overdiagnosis of chronic obstructive pulmonary disease. Am J Respir Crit Care Med. 2018;198(9):1130-1139. doi:10.1164/rccm.201804$0621 \mathrm{CI}$

13. StataCorp. Stata Statistical Software: release 15. 2017.

14. Micallef L, Rodgers P. eulerAPE: Drawing Area-proportional 3-Venn Diagrams Using Ellipses. PLoS ONE. 2014;9(7):e101717. doi:10.1371/journal.pone.0101717

15. Katsimigas A, Tupper O, Ulrik C. Opportunistic screening for COPD in primary care: a pooled analysis of 6,710 symptomatic smokers and ex-smokers. Int J COPD. 2019;14:1633-1638.

16. Johns DP, Pierce R. Spirometry: The Measurement and Interpretation of Ventilatory Function in Clinical Practice. Melbourne: The Thoracic Society of Australia and New Zealand; 2008.

17. Zammit C, Liddicoat H, Moonsie I, Makker H. Obesity and respiratory diseases. Int J Gen Med. 2010;3:335-343. doi:10.2147/IJGM.S11926

18. Poulain M, Doucet M, Major GC, et al. The effect of obesity on chronic respiratory diseases: pathophysiology and therapeutic strategies. CMAJ. 2006;174(9):1293-1299. doi:10.1503/cmaj.051299

19. Australian Institute of Health and Welfare (AIHW). Overweight and obesity: an interactive insight. Available from: https://www.aihw.gov. au/reports/overweight-obesity/overweight-and-obesity-an-interactiveinsight. Accessed September 26, 2019.

20. Liang J, Abramson MJ, Zwar NA, et al. Diagnosing COPD and supporting smoking cessation in general practice: evidence-practice gaps. Med J Aust. 2018;208(1):29-34. doi:10.5694/mja17.00664

21. Petrie K, Abramson MJ, Cross AJ, George J. Predicting life expectancy of older people using respiratory symptoms and smoking status: data from the Australian longitudinal study of ageing. Respirology. 2020;25(3):267-274. doi:10.1111/resp.13603

22. GINA/GOLD. Asthma COPD and asthma - COPD overlap syndrome. 2015. Available from: http://ginasthma.org/asthma-copdand-asthma-copd-overlap-syndrome-acos/. Accessed February 5, 2021. 
23. Collins BF, Feemster LC, Rinne ST, Au DH. Factors predictive of airflow obstruction among veterans with presumed empirical diagnosis and treatment of COPD. Chest. 2015;147(2):369-376. doi:10.1378/chest.14-0672

24. Walters JA, Walters EH, Nelson M, et al. Factors associated with misdiagnosis of COPD in primary care. Prim Care Respir J. 2011;20 (4):396. doi:10.4104/pcrj.2011.00039

25. Abad Arranz M, Morán-Rodríguez A, Mascarós Balaguer E, et al. Quantification of inaccurate diagnosis of COPD in primary care medicine: an analysis of the $\mathrm{COACH}$ clinical audit. Int $J$ Chron Obstruct Pulmon Dis. 2019;14:1187-1194. doi:10.2147/COPD. S199322

26. Vollmer WM, Gíslason P, Burney P, et al. Comparison of spirometry criteria for the diagnosis of COPD: results from the BOLD study. Eur Respir J. 2009;34(3):588-597. doi:10.1183/09031936.00164608

27. Eschenbacher WL. Airflow limitation and spirometry. In: Panos R, editor. COPD Clinical Perspectives. 2014.
28. Lamprecht B, Soriano JB, Studnicka M, et al. Determinants of underdiagnosis of COPD in national and international surveys. Chest. 2015;148(4):971-985. doi:10.1378/chest.14-2535

29. Australian Bureau of Statistics. National Health Survey: First Results, 2017-18 Vol ABS Cat no. 4364. 0.55.001. Canberra; 2018.

30. Le L, Johannessen A, Hardie J, et al. Prevalence and prognostic ability of the GOLD 2017 classification compared to the GOLD 2011 classification in a Norwegian COPD cohort. Int $J$ COPD. 2019;14:1639-1655.

31. Tejero E, Prats E, Casitas R, et al. Classification of airflow limitation based on Z-score underestimates mortality in patients with chronic obstructive pulmonary disease. Am J Respir Crit Care Med. 2017;196 (3):298-305. doi:10.1164/rccm.201611-2265OC

\section{Publish your work in this journal}

The International Journal of COPD is an international, peer-reviewed journal of therapeutics and pharmacology focusing on concise rapid reporting of clinical studies and reviews in COPD. Special focus is given to the pathophysiological processes underlying the disease, intervention programs, patient focused education, and self management protocols. This journal is indexed on PubMed Central, MedLine and CAS. The manuscript management system is completely online and includes a very quick and fair peer-review system, which is all easy to use. Visit http://www.dovepress.com/testimonials.php to read real quotes from published authors. 